\title{
Evaluation of Risk Factors for Vertebral Compression Fracture after Stereotactic Radiosurgery in Spinal Tumor Patients
}

\author{
Sang-Hyun Sung, Ung-Kyu Chang \\ Department of Neurosurgery, Korea Cancer Center Hospital, Korea Institute of Radiological and Medical Sciences, Seoul, Korea
}

Objective: Stereotactic radiosurgery (SRS) is an emerging treatment modality for malignant spinal tumors. After SRS, some patients suffered from pain aggravation due to development of vertebral compression fracture (VCF). In these cases, surgery should be considered.

Methods: This study consisted of 72 patients who underwent SRS due to spinal tumors. In them, whether post-SRS VCF developed or not was investigated. We retrospectively analyzed their medical records and radiological imaging data. VCF was diagnosed with X-ray and magnetic resonance imaging (MRI). The incidence, time to development and risk factors for VCF were investigated. Age, sex, whole vertebral body involvement rate, vertebral body osteolysis rate, pre-SRS spinal deformity, spinal instability neoplastic score (SINS), spinal canal encroachment, lesion level, and radiation dose were analyzed as potential risk factors. A multi-variate logistic regression model was used for statistical analysis.

Results: In our study population, VCF was observed in 26 patients (36\%). The mean time to VCF development was 1.5 months. Using uni-variate analyses, the significant risk factors were pre-SRS spinal deformity, SINS, vertebral body osteolysis rate, and whole vertebral body involvement rate. However, using multi-variate analyses, the only significant risk factor was vertebral body osteolysis rate. The patients whose vertebral body was destroyed by more than $60 \%$ showed an 8.4 times higher risk of VCF than those who had vertebral body destruction of less than $60 \%(p=0.016)$.

Conclusion: The most significant prognostic factor for post-SRS VCF was vertebral body osteolysis rate, rather than whole vertebral body involvement rate. When more than $60 \%$ of the vertebral body was destroyed, the risk of VCF or spinal deformity was high.

Key Words: Stereotactic radiosurgery $\cdot$ Compression fracture $\cdot$ Sequelae

\section{INTRODUCTION}

In the treatment of spinal tumors, radiotherapy is considered as the essential treatment modality ${ }^{5}$. Recently, stereotactic radiosurgery (SRS) has been developed and widely used in the field of spine tumor treatment. One of the major complications associated with SRS is vertebral compression fracture $(\mathrm{VCF})^{3,7,11)}$. In cases of osteolytic spinal tumors, vertebral body and pedicle are eroded by tumor mass and the remaining bony structures must withstand spinal loading. When radia-

- Received: August 4, 2014 • Revised: August 25, 2014

- Accepted: September 1, 2014

Corresponding Author: Ung-Kyu Chang, MD, PhD

Department of Neurosurgery, Korea Cancer Center Hospital, Korea Institute of Radiological and Medical Sciences, 215-4 Gongreung-dong, Nowon-gu, Seoul 139-706, Korea

Tel: +82-2-970-1238, Fax: +82-2-970-1267, E-mail: cuk@kcch.re.kr $\otimes$ This is an Open Access article distributed under the terms of the Creative Commons Attribution Non-Commercial License (http://creativecommons.org/ licenses/by-nc/3.0/) which permits unrestricted non-commercial use, distribution, and reproduction in any medium, provided the original work is properly cited. tion is delivered to spinal tumor and the remaining vertebral body, soft mass shrinkage and bony necrosis can develop, which leads to VCF and the resultant kyphotic deformity ${ }^{10}$. The risk of VCF following SRS has been investigated in several studies. A prospective trial by Rose et al. revealed that 39\% of treated vertebral bodies showed new or progressing fractures, in which larger, lytic lesions located in the lower spine presented a high risk ${ }^{11)}$. Larger radiation dose, previous spinal deformity, histology, age of more than 55 years and vertebral body involvement by at least $40-60 \%$ were suggested as significant risk factors ${ }^{3,7,12,13)}$. In cases of significant VCF, vertebroplasty or stabilization surgery should be considered.

The Spine Oncology Study Group (SOSG) defines spine instability as the "loss of spinal integrity as a result of a neoplastic process that is associated with movement-related pain, symptomatic or progressive deformity and/or neural compromise under physiological loads" ${ }^{8)}$. The SOSG developed the Spinal Instability Neoplastic Score (SINS) in 2011,9. Under this classification system, tumor-related instability is assessed by adding together six individual component scores: spine location, 
mechanical pain, lesion bone type (lytic or blastic), radiographic alignment, vertebral body collapse, and posterolateral involvement of the spinal elements (Table 1). The minimum score is 0 , and the maximum is 18 . A score of 0-6 means stable condition, 7-12 denotes indeterminate (possibly impending) instability, and 13-18 instability. A surgical consultation is recommended for patients with SINS greater than 7 .

We investigated the incidence and time to development of VCF following SRS. Predictive risk factors of VCF including overall score of SINS system were also analyzed.

\section{MATERIALS AND METHODS}

The study cohort was 72 patients with malignant spinal column tumors, who underwent SRS (Table 2). Tumor histology was described in Table 3. The mean age of the participants was 51 years (range, 19-78 years). The ratio of males to females was 42:30. Regarding the lesion level, 17 tumors were located in the cervical region, 26 in the thoracic region, and 29 in the lumbar spine. Pre-SRS deformities such as compression fracture or kyphosis were present in 8 patients. Spinal canal encroachment by tumor mass was observed in 22 patients. SINS ranged from 2 to 15 (mean score $=8$ ). A modified Weins-

Table 1. Spinal instability neoplastic scoring (SINS) system.

\begin{tabular}{|c|c|c|}
\hline SINS component & Description & Score \\
\hline \multirow[t]{4}{*}{ Location } & $\begin{array}{l}\text { Junctional (occiput-C2, C7-T2, } \\
\text { T11-L1, L5-S1) }\end{array}$ & 3 \\
\hline & Mobile spine (C3-C6, L2-L4) & 2 \\
\hline & Semi-rigid (T3-T10) & 1 \\
\hline & Rigid (S2-S5) & 0 \\
\hline \multirow[t]{3}{*}{ Pain } & Yes & 3 \\
\hline & Occasional pain but not mechanical & 1 \\
\hline & Pain-free lesion & 0 \\
\hline \multirow[t]{3}{*}{ Bone lesion } & Lytic & 2 \\
\hline & Mixed (lytic/blastic) & 1 \\
\hline & Blastic & 0 \\
\hline Radiographic & Subluxation/translation & 4 \\
\hline \multirow[t]{2}{*}{ spinal alignment } & Kyphosis/scoliosis & 2 \\
\hline & Normal & 0 \\
\hline Vertebral body & $50 \%$ collapse & 3 \\
\hline \multirow[t]{3}{*}{ collapse } & $50 \%$ collapse & 2 \\
\hline & No collapse with $50 \%$ body involved & 1 \\
\hline & None of the above & 0 \\
\hline Posterolateral & Bilateral & 3 \\
\hline involvement of & Unilateral & 1 \\
\hline spinal elements & None of the above & 0 \\
\hline
\end{tabular}

tein-Boriani-Biagini (WBB) classification system was adopted to assess the extent of tumor infiltration in the whole vertebral body $^{4)}$ (Fig. 1A). This measure was developed specifically to stage in patients with primary spine tumors. In this system, the whole vertebral body including posterior element is divided into 12 sectors. The sectors were numbered counterclo-

Table 2. Summary of patient characteristics

\begin{tabular}{|c|c|}
\hline Variable & Case numbers \\
\hline Total patients & 72 \\
\hline \multicolumn{2}{|l|}{ Sex } \\
\hline M & 42 \\
\hline$F$ & 30 \\
\hline \multicolumn{2}{|l|}{ Age } \\
\hline$<51$ & 31 \\
\hline$\geq 51$ & 41 \\
\hline \multicolumn{2}{|l|}{ Lesion level } \\
\hline Cenvical & 17 \\
\hline Thoracic & 26 \\
\hline lumbar & 29 \\
\hline \multicolumn{2}{|c|}{ Pre-SRS deformity } \\
\hline Yes & 8 \\
\hline No & 64 \\
\hline \multicolumn{2}{|l|}{ SINS } \\
\hline $0-5$ & 12 \\
\hline $6-10$ & 50 \\
\hline $11-1$ & 10 \\
\hline \multicolumn{2}{|c|}{ VB osteolysis rate } \\
\hline$<60 \%$ & 34 \\
\hline$\geq 60 \%$ & 38 \\
\hline \multicolumn{2}{|c|}{ Whole VB involvement rate } \\
\hline$<40 \%$ & 39 \\
\hline$\geq 40 \%$ & 33 \\
\hline \multicolumn{2}{|c|}{ Irradiation dose } \\
\hline$<21 \mathrm{~Gy}$ & 50 \\
\hline$\geq 21 \mathrm{~Gy}$ & 22 \\
\hline \multicolumn{2}{|c|}{ Spinal canal encroachment } \\
\hline Yes & 22 \\
\hline No & 50 \\
\hline
\end{tabular}

SINS, Spinal instability neoplastic score; VB, vertebral body; SRS, stereotactic radiosurgery.

Table 3. Tumor histology

\begin{tabular}{lc}
\hline \hline Histology & Number of cases \\
\hline Breast cancer & 15 \\
Lung cancer & 11 \\
Sarcoma & 11 \\
Hepatocellular carcinoma & 8 \\
Thyroid cancer & 8 \\
Renal cell carcinoma & 6 \\
Others & 13 \\
Total patients & 72 \\
\hline
\end{tabular}




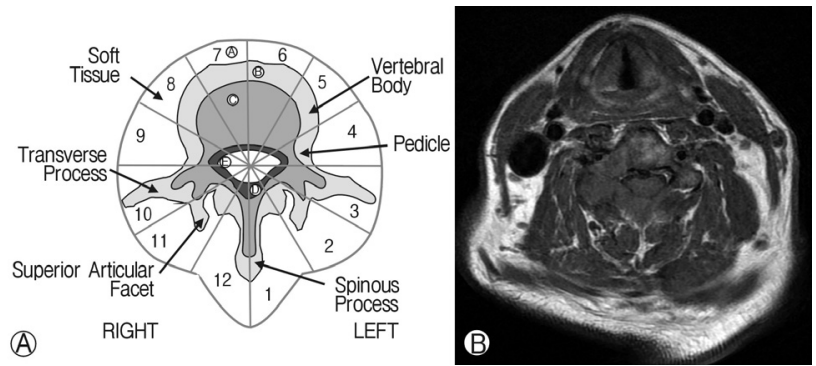

Fig. 1. (A) Modified Weinstein-Boriani-Biagini (WBB) classification system. In this system, the whole vertebral body including the posterior element was divided into 12 sectors. (B) The extent of tumor infiltration was determined using T1-axial MRI. The tumor was shown to infiltrate both laminae, right pedicle and right vertebral body, which corresponded to sectors 8-12, 1, and 2. In this case, the whole vertebral body involvement rate was $7 / 12$ (66\%). The sectors of vertebral body excluding posterior element were 4-9 sectors. Tumor was present only in sector 8 and 9. In this case, vertebral body osteolysis rate was $2 / 6(33 \%)$.

ckwise. The rate of whole vertebral body involvement was defined as the ratio of the number of tumor-infiltrated sectors to whole sectors (sector 1-12). Vertebral body osteolysis rate is defined as the ratio of the number of tumor-infiltrated sectors to sectors 4-9. To evaluate the extent of tumor infiltration, T1 weighted axial MRI was used. For example, Fig. 1B shows a tumor infiltrating both laminae, the right pedicle and right vertebral body, which correspond to sectors $8-12,1$, and 2 . The number of involved sectors was 7 . The rate of whole vertebral body involvement was 7/12 (66\%). The sectors of vertebral body excluding posterior element are 4-9. In Fig. 2B, tumor was present in sector 8 and 9. In this case, vertebral body osteolysis rate was 2/6 (33\%).

SRS was performed as an outpatient procedure in most cases. Radiation doses ranged from 18 to $45 \mathrm{~Gy}$ with 1-5 fractionations. When these values were calculated as single equivalent SRS doses, they ranged from 18 Gy to $26 \mathrm{~Gy}$ (mean $=21 \mathrm{~Gy}$ ). After SRS, patients were assessed with regular follow-up for pain status, neurological status and radiological response. X-rays were checked every month for 3 months and MRI was used to evaluate radiological response. VCF was defined as development of a new fracture or progression of pre-existent fractures with relevant symptoms.

Analyzed risk factors of VCF were age, sex, whole vertebral body involvement rate, vertebral body osteolysis rate, pre-SRS spinal deformity, spinal instability neoplastic score (SINS), spinal canal encroachment, lesion level, and radiation dose. Continuous variables such as age, whole spine involvement rate, vertebral body osteolysis rate, SINS and radiation dose were categorized as two groups (below mean and above mean). Pre-SRS spinal deformity and spinal encroachment were also

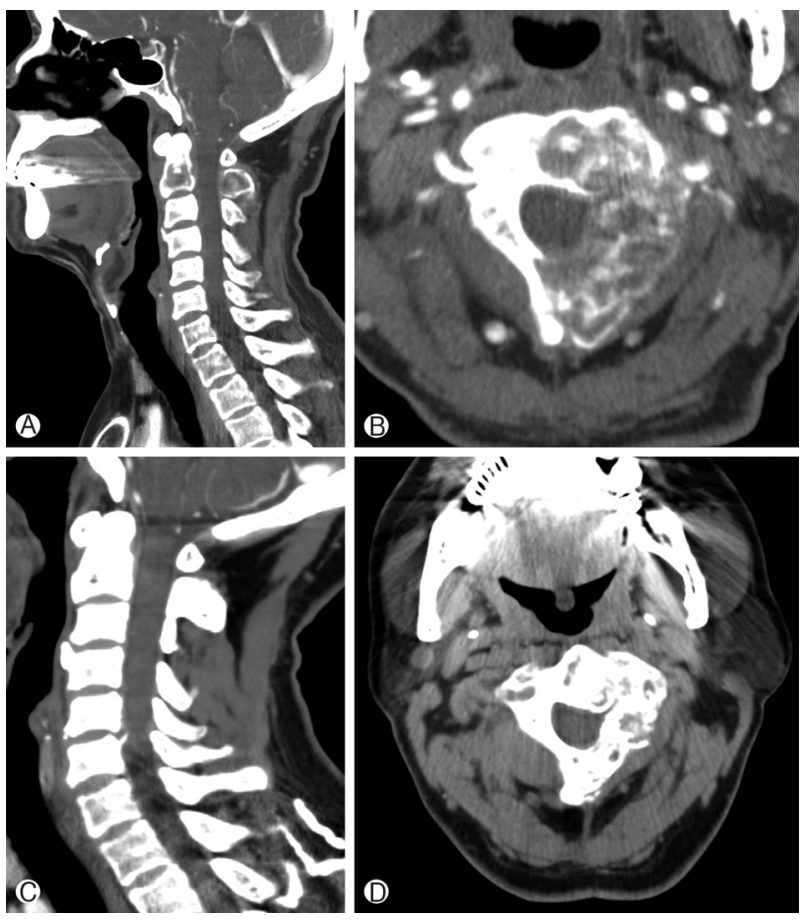

Fig. 2. (A) Pre-SRS computed tomography (CT) sagittal view, showing an osteolytic lesion infiltrating the C2 vertebral body and spinous process. (B) Pre-SRS axial CT image, showing a tumor mass eroding the left side of the C2 vertebral body, left pedicle, and left lamina. The spinal instability neoplastic score was 10 points. Whole vertebral body involvement rate was $67 \%$ and vertebral body osteolysis rate was 65\%. (C) Post-SRS CT sagittal view at 24 months follow-up. Cenvical spine alignment was good and bone healing was seen at the site of osteolyitc lesion. (D) Post-SRS CT axial view at 24 months follow-up. Bone remodeling was apparent.

analyzed as categorical factors, which mean the presence or absence of the factors. A multi-variate logistic regression model was used for the statistical analysis. Resulting $\mathrm{p}$ values were considered statistically significant at $<0.05$.

\section{RESULTS}

We observed 26 fractures (26 of 72 patients, 36\%). The mean follow-up for the study population was 11 months (range, 3-24 months). The mean time to fracture for those patients who developed VCF was 1.5 months (range, 0.3-3.5 months). According to uni-variate analyses, whole vertebral body involvement rate $(p=0.007)$, pre-SRS deformity $(p=0.013)$, vertebral body osteolysis rate $(p=0.001)$, and SINS $(p=0.002)$ were significant factors (Table 4). However, multi-variate analyses confirmed that only vertebral body osteolysis rate was predictive of VCF development $(p=0.016$ ). Patients whose verte- 
bral body was destroyed by more than $60 \%$ showed an 8.4 times higher risk of VCF than those who had vertebral body destruction of less than $60 \%$. Pre-SRS deformity $(p=0.31)$, SINS $(p=0.07)$, and whole vertebral body involvement rate $(p$ $=0.8$ ) were not significant predictive factors. Ten patients underwent percutaneous vertebroplasty and 5 underwent fusion surgery to treat pain associated with post-SRS VCF.

Two illustrative cases are presented in this report. Patient
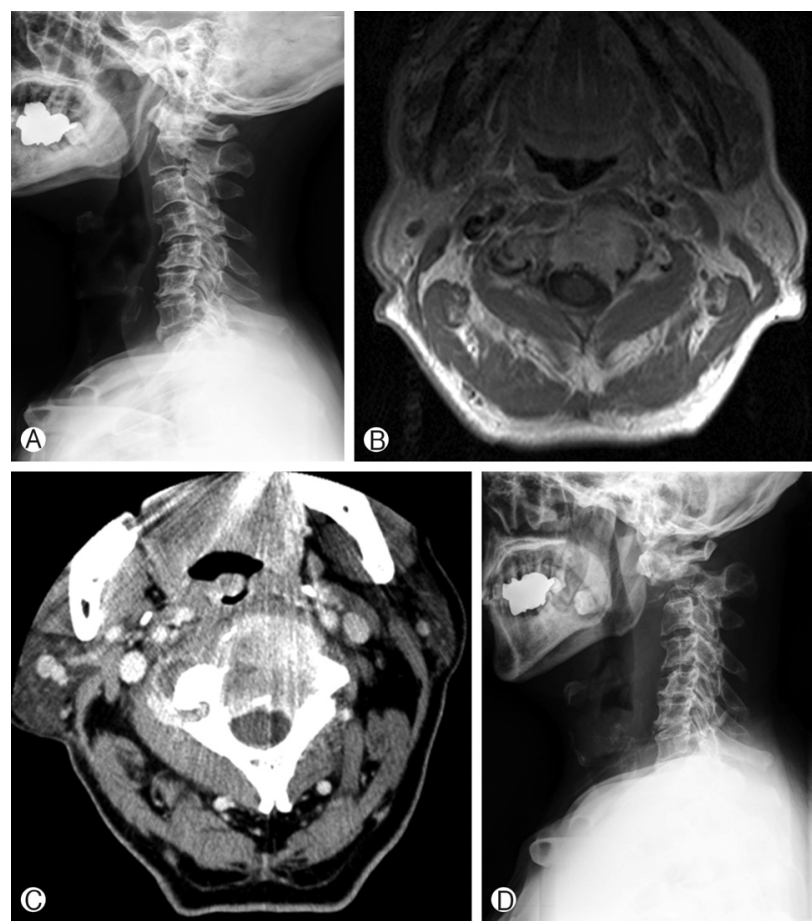

Fig. 3. (A) Cervical spine lateral view of a patient with renal cell cancer C2 metastasis. Pre-SRS kyphotic deformity is present. (B) Pre-SRS MRI axial view showing the tumor mass infiltrating the C2 vertebral body. (C) Pre-SRS CT axial image. Spinal instability neoplastic score was 10. Whole vertebral body involvement rate was $67 \%$, but the vertebral body osteolysis rate was $90 \%$. (D) Post- SRS cenvical spine lateral view. A C2 compression fracture developed and cervical kyphosis progressed.
1, a 46-year-old man, complained of neck pain. MRI showed a metastatic spine tumor infiltrating $\mathrm{C} 2$ vertebral body, left pedicle and left lamina (Fig. 2). For this patient, the SINS was 10 and the vertebral body osteolysis rate was $65 \%$. The whole vertebral body involvement rate was $67 \%$. SRS was performed with 32 Gy in 4 fractions. After SRS, his pain improved and VCF was not detected at 24 months follow-up. In case 2, the metastatic spine tumor involved the vertebral body only (Fig. 3). The SINS was 10 and the whole vertebral body involvement rate was $67 \%$. However, the vertebral body osteolysis rate was 90\%. In addition, pre-SRS kyphosis was present. Following SRS, the neck pain aggravated and follow-up X-ray showed progression of kyphosis at the C2-3 level.

\section{DISCUSSION}

SRS is an emerging treatment for spinal tumors and is widely applied in clinical setting. VCF following SRS is increasingly recognized as a significant and common adverse event ${ }^{13)}$. Although the risk of VCF is approximately $5 \%$ after conventional radiotherapy, crude risk estimates for VCF after spinal SRS range from $11 \%$ to $39 \%{ }^{13)}$. The incidence of VCF reported after spinal SRS is variable depending on the reporting authors. The first report on post-SRS VCF was by Rose et al., who reported VCF in 27 (39\%) of 71 sited treated with SRS ${ }^{11)}$. They treated patients with high-dose single fraction SRS (median dose-24 Gy in one fraction). Authors from MD Anderson Cancer Center investigated 127 vertebral bodies of 93 patients, and reported a $20 \%$ risk of VCF with a threemonth median time to $\mathrm{VCF}^{3)}$. Cunha et al. analyzed 167 spinal segments in 90 patients treated with SRS and reported an actuarial VCF rate of $12.7 \%$ at 1 year post-SRS with a mean time to VCF of 3 months $^{7}$. In this study, patients with SRS of $20 \mathrm{~Gy}$ or greater were at a higher risk of VCF. In a recent study by Sahgal et al., the approximate risk was 14\%, and the 1-year cumulative incidence was $12.35 \%{ }^{12)}$. In that report, it was clear

Table 4. Uni-variate and multi-variate analyses of predictors for post-SRS VCF

\begin{tabular}{|c|c|c|c|}
\hline Factors analyzed & Classified group & Univariate $\mathrm{p}$-value/Hazard ratio & Multivariate p-value/Hazard Ratio \\
\hline Age & $<51$ vs. $\geq 51$ & 0.854 & \\
\hline Sex & Male vs. female & 0.375 & \\
\hline Location & Cervical vs. thoracic vs. lumbar & 0.091 & \\
\hline Whole VB involvement rate & $<40 \%$ vs $\geq 40 \%$ & $0.007 / 4.000$ & 0.891 \\
\hline Pre-SRS deformity & Yes vs. no & $0.013 / 15.400$ & 0.307 \\
\hline VB osteolysis rate & $<61 \%$ vs. $\geq 61 \%$ & $<0.001 / 11.500$ & $0.016 / 8.474$ \\
\hline SINS & $0-5$ vs. $6-10$ vs. $11-15$ & $0.002 / 10.900$ & 0.203 \\
\hline Irradiation dose & $<21$ Gy vs. $\geq 21$ Gy & 0.510 & \\
\hline Spinal canal enchroachment & Yes vs. no & 0.357 & \\
\hline
\end{tabular}

VB, vertebral body; SRS, stereotactic radiosurgery; SINS, Spinal instability neoplastic score; VCF, vertebral compression fracture. 
that as the radiation dose increases, the risk of VCF significantly increased. The incidence of VCF was 39\% in the group with radiation doses of $>24 \mathrm{~Gy}$. These results explain the previously reported 39\% risk of VCF by Rose et al. The risk of late normal tissue adverse effects increases as the radiation dose increases. Similar findings that doses $>20 \mathrm{~Gy}$ are associated with a greater risk of radiation necrosis have been obtained from brain radiosurgery $^{144}$. In the present study, the incidence of VCF was 36\%, which was higher than the risk reported by other authors. And it was not different depending on the radiation dose. The reason was thought to be high radiation dose (mean=21 Gy). Several reports on post-SRS VCF suggest that if the radiation dose is reduced, the adverse events associated with VCF can be avoided.

The present findings suggested whole vertebral body involvement rate, pre-SRS deformity, vertebral body osteolysis rate, and SINS as risk factors for VCF, using uni-variate analyses. Of these factors, vertebral body osteolysis rate was the only significant factor using multi-variate analyses. This finding is consistent with those of other studies, ${ }^{7,11,12}$. Boehling et al. investigated 127 vertebral bodies from 93 patients. In their study, risk factors of VCF included age $>55$ years, a preexisting fracture, and baseline mechanical pain ${ }^{3}$. A report by Cunha et al. states that the presence of kyphotic/scoliotic deformity, presence of a lytic tumor, specific histology (lung and hepatocelluar carcinoma metastasis), and radiation dose of $\geq 20$ Gy were predictive factors of VCF. Sahgal et al. investigated the risk factors of VCF using six criteria of SINS; location, presence of mechanical pain, bone lesion type, alignment, baseline VCF, and posterior element involvement. Among six criteria, baseline VCF, bone lesion type (lytic tumor), and malalignment were significant factors. However, the overall score was not predictive, which is compatible with our present results. Another study by Cunha et al., which also evaluated SINS criteria as predictive risk factor of VCF, revealed that the presence of a lytic tumor, and the presence of malalignment were significant and baseline VCF was excluded ${ }^{7}$. The most frequently cited risk factor was the presence of a lytic tumor ${ }^{3,7,11,12)}$. Rose et al.'s study investigated the risk of fracture depending on the degree of the vertebral body involvement rate. Classification according to the amount of the vertebral body occupied by the lesion resulted in a significantly different fracture incidence. The risk of VCF was $22 \%$ in the group with $1-20 \%$ vertebral body involvement, $58 \%$ in the group with $2140 \%$ involvement, $86 \%$ in the patients with $41-60 \%$ involvement, and $83 \%$ in the patients with $61-80 \%$ involvement. There were a significant difference in the risk of VCF between the group with vertebral body involvement $<40 \%$ and the group with vertebral body involvement $>40 \%$, which is compatible with the results of the present study. Our data indicate that patients with vertebral body osteolysis rates more than $60 \%$ showed higher risk of VCF than those with rates less than $60 \%$.

Osteoradionecrosis, which lead to both healthy vertebral bone and tumor tissue being replaced with friable necrotic tissue, is the proposed mechanism of SRS induced VCF. This process causes further mechanical compromise and an increased likelihood of fracture ${ }^{13)}$. Osteoradionecrosis has been defined as a slow-healing radiation-induced ischemic necrosis of the bone tissue. The mechanism of osteoradionecrosis is thought to relate to radiation therapy by the production of a hypoxic, hypocellular, and hypovascular environment in which the basic metabolic demands for cellular survival cannot be met ${ }^{15}$. The onset of osteoradionecrosis has been described to range from 6 months to 3 years following treatment, and may even occur as late as 25 years after radiation therapy. In SRS planning, the entire vertebral body is included in the target volume, even if focal involvement was diagnosed ${ }^{6}$. Recent data have confirmed a great risk of failure if the target is restricted to a visible tumor within the vertebral body. Therefore, both the healthy trabecular bone and tumor tissue are exposed to the high radiation dose. Al-Omair et al. reported biopsy result in SRS-induced VCF cases ${ }^{1}$. Histopathological analysis showed markedly thinned bone trabeculae, and the bone marrow was completely replaced by either dense fibrous tissue with focal lymphocytic inflammation or loose paucicellular fibrous tissue. Bone is a complex two-phase composite substance containing mineral (hydroxyapatite) and organic (collagen) components ${ }^{13}$. The mineral component gives strength and stiffness to bone tissue whereas the collagen part gives bone ductility, increasing the tissue toughness. Decreases in the strength, modulus, and toughness of the collagen network are associated with decline in bone strength. A high radiation dose damages collagen, which reduces bone toughness. The mechanism of radiation-associated collagen damage might be an increase in the cross-link ratios ${ }^{2)}$. Ultimately, exposure to radiation progressively degrades the strength, ductility, and toughness of the bone tissue ${ }^{2)}$.

The management of VCF consisted of conservative treatment, bone cement augmentation, or surgical stabilization. The proportion of patients who underwent vertebroplasty or surgery was $3 / 27^{11)}, 10 / 25^{3)}$, and $9 / 19^{7}$. Of these patients, percutaneous cement augmentation procedures were used more commonly (77\%) than spinal stabilization surgery (23\%). The choice of surgical intervention is determined by radiological finding of the spine. For example, VCF without kyphosis and without substantial posterior element involvement can be treated with a percutaneous cement augmentation procedure alone. By contrast, patients with kyphotic deformity or translation instability generally need a combination of vertebral augmentation and pedicle screw reconstruction. Additionally, the systemic status of the patient, tumor pathology, and extent of metastatic bur- 
den need to be carefully considered when deciding on surgical options. Of the patients in this study, 15 out of 26 patients with VCF were treated with vertebroplasty (10 patients) and stabilization operation (5 patients).

\section{CONCLUSION}

VCF was a relatively common adverse event after spinal SRS (36\%). Vertebral body osteolysis rate more than 60\% was the only significant risk factor for post-SRS VCF demonstrated by our findings. VCF can be clinically significant, resulting in mechanical instability. Surgical intervention should be considered in the patients with post-SRS VCF.

\section{REFERENCES}

1. Al-Omair A, Smith R, Kiehl TR, Lao L, Yu E, Massicotte EM, et al: Radiation-induced vertebral compression fracture following spine stereotactic radiosurgery: Clinicopathological correlation. J Neurosurg Spine 18:430-435, 2013

2. Barth HD, Zimmermann EA, Schaible E, Tang SY, Alliston T, Ritchie RO: Characterization of the effects of X-ray irradiation on the hierarchical structure and mechanical properties of human cortical bone. Biomaterials 32:8892-8904, 2011

3. Boehling NS, Grosshans DR, Allen PK, McAleer MF, Burton AW, Azeem S, et al: Vertebral compression fracture risk after stereotactic body radiotherapy for spinal metastases. J Neurosurg Spine 16:379-386, 2012

4. Chan P, Boriani S, Fourney DR, Biagini R, Dekutoski MB, Fehlings MG, et al: An assessment of the reliability of the enneking and weinstein-boriani-biagini classifications for staging of primary spinal tumors by the spine oncology study group. Spine (Phila Pa 1976) 34:384-391, 2009

5. Chow E, Harris K, Fan G, Tsao M, Sze WM: Palliative radiotherapy trials for bone metastases: A systematic review. J Clin Oncol 25:1423-1436, 2007

6. Cox BW, Spratt DE, Lovelock M, Bilsky MH, Lis E, Ryu S, et al: International spine radiosurgery consortium consensus guidelines for target volume definition in spinal stereotactic radiosurgery. Int J Radiat Oncol Biol Phys 83:e597-605, 2012

7. Cunha MV, Al-Omair A, Atenafu EG, Masucci GL, Letourneau D, Korol R, et al: Vertebral compression fracture (VCF) after spine stereotactic body radiation therapy (SBRT): Analysis of predictive factors. Int J Radiat Oncol Biol Phys 84:e343-9, 2012

8. Fisher CG, DiPaola CP, Ryken TC, Bilsky MH, Shaffrey CI, Berven $\mathrm{SH}$, et al: A novel classification system for spinal instability in neoplastic disease: An evidence-based approach and expert consensus from the spine oncology study group. Spine (Phila Pa 1976) 35:E1221-9, 2010

9. Fourney DR, Frangou EM, Ryken TC, Dipaola CP, Shaffrey CI, Berven SH, et al: Spinal instability neoplastic score: An analysis of reliability and validity from the spine oncology study group. J Clin Oncol 29:3072-3077, 2011

10. Gerszten PC, Germanwala A, Burton SA, Welch WC, Ozhasoglu C, Vogel WJ: Combination kyphoplasty and spinal radiosurgery: A new treatment paradigm for pathological fractures. J Neurosurg Spine 3:296-301, 2005

11. Rose PS, Laufer I, Boland PJ, Hanover A, Bilsky MH, Yamada $\mathrm{J}$, et al: Risk of fracture after single fraction image-guided intensity-modulated radiation therapy to spinal metastases. J Clin Oncol 27:5075-5079, 2009

12. Sahgal A, Atenafu EG, Chao S, Al-Omair A, Boehling N, Balagamwala $\mathrm{EH}$, et al: Vertebral compression fracture after spine stereotactic body radiotherapy: A multi-institutional analysis with a focus on radiation dose and the spinal instability neoplastic score. J Clin Oncol 31:3426-3431, 2013

13. Sahgal A, Whyne CM, Ma L, Larson DA, Fehlings MG: Vertebral compression fracture after stereotactic body radiotherapy for spinal metastases. Lancet Oncol 14:e310-20, 2013

14. Shaw E, Scott C, Souhami L, Dinapoli R, Kline R, Loeffler J, et al: Single dose radiosurgical treatment of recurrent previously irradiated primary brain tumors and brain metastases: Final report of RTOG protocol 90-05. Int J Radiat Oncol Biol Phys 47:291298, 2000

15. Zhuang Q, Zhang Z, Fu H, He J, He Y: Does radiation-induced fibrosis have an important role in pathophysiology of the osteoradionecrosis of jaw? Med Hypotheses 77:63-65, 2011 